\title{
AVALIAÇÃO DOS RESULTADOS DA CIRURGIA CONSERVADORA DO CÂNCER DA LARINGE
}

\author{
RESULTS OF CONSERVATIVE SURGERY IN LARYNX CARCINOMA
}

\author{
José Luís Braga de Aquino, TCBC-SP ${ }^{1}$ \\ José Gonzaga Teixeira Camargo ${ }^{1}$ \\ Claudiney Cândido Costa ${ }^{1}$ \\ Maria Beatriz Nogueira Paschoal ${ }^{1}$ \\ José Francisco Salles Chagas ${ }^{2}$
}

\begin{abstract}
RESUMO: Objetivo: Avaliar retrospectivamente os resultados de pacientes submetidos a laringectomias parciais por carcinoma epidermóide de laringe no serviço do HMCP. Métodos: Dos doentes estudados, 28 eram do sexo masculino $(93,5 \%)$ e dois do feminino (6,5\%) com idade variável de 42 a 66 anos, com média de 58 anos. Em relação ao procedimento cirúrgico, este foi variável, dependendo do local da extensão tumoral e do estádio. A técnica mais utilizada foi a laringectomia frontolateral em 12 pacientes (40\%), seguido pela laringectomia vertical em oito (26,6\%), laringectomia supraglótica em quatro $(13,3 \%)$, laringectomia supracricóidea em quatro (13,3\%), laringectomia near-total em um (3,3\%) e cordectomia em um (3,3\%). Resultados: $\mathrm{Na}$ avaliação pós-operatória precoce, três pacientes (10\%) apresentaram complicações evidenciadas por fístula faringocutâneas em dois, com boa evolução após tratamento conservador, e pneumotórax no paciente restante com boa evolução após drenagem do tórax. No que se refere à avaliação da deglutição, cinco pacientes $(16,6 \%)$ apresentaram dificuldade de ingestão oral devido a aspirações recorrentes com tempo variável de 45 a 180 dias (média - 65 dias), o que obrigou a um paciente ser submetido à totalização da laringectomia devido a pneumonias recorrentes. Na avaliação tardia, de seis a 125 meses e média de 29 meses, quatro pacientes $(13,3 \%)$ apresentaram recidiva locorregional, sendo dois submetidos à laringectomia vertical, um à frontolateral e o restante à supracricóidea. Em todos foram realizadas totalização e/ou radioterapia, e dois evoluíram a óbito pela doença. Vinte e quatro pacientes (84\%) apresentaram preservação da voz. Conclusão: Assim, os autores concluem que a cirurgia conservadora da laringe proporciona bom controle da doença e com sobrevida aceitável, já que $90 \%$ dos pacientes estudados estão vivos e sem doença até o final da avaliação. Além disso, apresentam melhor qualidade de vida já que a maioria dos pacientes demonstrou voz e ingestão oral bem satisfatórias.
\end{abstract}

Descritores: Laringe; Câncer; Laringectomia.

\section{INTRODUÇÃO}

Desde o final do século XIX a laringectomia parcial vem sendo introduzida gradualmente no contexto técnico da cirurgia de cabeça e pescoço ${ }^{1}$.

Atualmente, muitos autores concordam que as técnicas de laringectomia parcial possibilitam, em casos selecionados, o controle da lesão primária, além de proporcionar qualidade de voz satisfatória, baixo índice de complicações e, na maioria das vezes, mantém o paciente sem traqueostomia definitiva ${ }^{1-16}$.

Desta maneira, a cirurgia conservadora da laringe tem duas grandes vantagens em relação à laringectomia total, pois a via aérea normal permanece intacta e a função glótica fica comprometida apenas parcialmente.

Outro fato que se discute atualmente é se o câncer glótico nas fases iniciais seria mais bem tratado por radioterapia ou cirurgia conservadora, seja aberta ou por via

1. Cirurgiões do Serviço de Cirurgia de Cabeça e Pescoço do HCMP - PUC.

2. Chefe do Serviço de Cirurgia de Cabeça e Pescoço do HMCP-PUC.

Recebido em 02/08/2001.

Aceito para publicação em 22/11/2001.

Trabalho realizado no Serviço de Cirurgia de Cabeça e Pescoço do Hospital e Maternidade Celso Pierro - PUC-Campinas (HMCP-PUC). 
endoscópica ${ }^{11,12,14,15}$. A cirurgia aberta é obviamente mais invasiva que a radioterapia, não sendo incomum o comprometimento parcial da deglutição e respiração. Por sua vez, vários autores têm demonstrado que a laringectomia parcial, com ou sem reconstrução, proporciona maior controle do tumor e maior taxa de sobrevida ${ }^{11,13,14}$.

Assim, o objetivo deste estudo é avaliar de maneira retrospectiva a eficácia da cirurgia conservadora em uma série de pacientes com carcinoma epidermóide do laringe.

\section{MÉTODO}

No período de janeiro de 1989 a dezembro de 1998, no Serviço de Cirurgia de Cabeça e Pescoço dos Hospitais Celso Pierro - PUC-Campinas e Heliópolis - São Paulo, foram estudados 30 pacientes portadores de carcinoma epidermóide da laringe, submetidos à cirurgia conservadora. Vinte e oito eram do sexo masculino $(93,5 \%)$ e dois do feminino (6,5\%), com idades variando de 42 a 76 anos (média de 58 anos). Quanto ao estádio, 14 pacientes eram T1N0, oito T2N0, três T2N1 e cinco T3N0. Em todos os pacientes foi realizado procedimento cirúrgico conservador, sendo o mesmo variável na dependência do local, da extensão tumoral e do estádio. A técnica mais utilizada foi a laringectomia frontolateral em 12 pacientes (Tabela 1).

Dos oito pacientes submetidos à hemilaringectomia vertical, em sete a reconstrução foi com retalho miocutâneo de músculo platisma e um com retalho de músculo trapézio.

Em cinco pacientes foi realizado esvaziamento cervical, e três tiveram esvaziamento radical unilateral por apresentarem adenopatia unilateral, complementando a laringectomia vertical em um e a laringectomia supraglótica em dois pacientes. Nos dois pacientes restantes, o esvaziamento foi jugular bilateral em lesões supraglóticas, apesar da ausência clínica de adenopatia metastática.

Tabela 1

Procedimentos cirúrgicos

\begin{tabular}{lcc}
\hline Técnica cirúrgica & Número & $\%$ \\
\hline Laringectomia frontolateral & 12 & 40 \\
Hemilaringectomia vertical & 08 & 26,6 \\
Laringectomia horizontal supraglótica & 04 & 13,3 \\
Laringectomia supracricóide & 04 & 13,3 \\
Cordectomia & 01 & 3,3 \\
Laringectomia near-total & 01 & 3,3 \\
\hline
\end{tabular}

\section{RESULTADOS}

Em relação às complicações, em avaliação realizada nos primeiros 30 dias de pós-operatório, dois pacientes $(6,6 \%)$ apresentaram fístula faringocutânea, um após laringectomia supraglótica e outro pós-laringectomia vertical, tendo boa evolução com terapêutica conservadora. Um paciente $(3,3 \%)$, submetido à ressecção pela técnica fron- tolateral, apresentou pneumotórax no terceiro dia de pósoperatório, tendo boa evolução após drenagem torácica.

No que concerne à deglutição, nos 17 pacientes estudados dos grupos da ressecção vertical, supraglótica, supracricóidea e near-total, cinco pacientes $(29,4 \%)$ apresentaram aspiração durante o ato de deglutição, sendo dois do grupo da supraglótica, dois da supracricóidea e um da vertical. Um dos pacientes do grupo da supraglótica com 60 dias de pós-operatório, por apresentar pneumonias recorrentes, foi submetido à totalização da laringectomia; o outro paciente desse grupo com 45 dias de pós-operatório começou a diminuir a frequiência de aspirações e a partir do $70^{\circ}$ dia iniciou deglutição satisfatória. Um paciente do grupo da supracricóidea conseguiu boa deglutição sem aspiração a partir do $60^{\circ}$ dia e o outro desse mesmo grupo permaneceu com sonda nasogástrica por mais ou menos seis meses, quando então começou a deglutir por via oral com pequenas aspirações. O paciente restante, pertencente ao grupo da ressecção vertical, voltou a apresentar deglutição normal com 45 dias.

No que se refere à decanulação, três pacientes (10\%) não puderam ser decanulados, um por se tratar de laringectomia near-total, outro submetido à laringectomia supracricóidea e outro por laringectomia vertical.

$\mathrm{Na}$ avaliação tardia de seis a 125 meses (média 29 meses) nos doentes submetidos à ressecção frontolateral, em um $(8,3 \%)$ houve recidiva com 23 meses de pós-operatório. Esse paciente foi submetido à radioterapia, mas com óbito após três meses por doença locorregional.

No grupo submetido à ressecção vertical, dois pacientes (25\%) apresentaram recidiva, e um foi a óbito com 12 meses por recidiva regional e o outro submetido á totalização por recidiva local com boa evolução até a última avaliação. Um paciente faleceu um ano e meio após a cirurgia por problemas cardiovasculares, sem recidiva.

No grupo submetido à ressecção supracricóide, um paciente (25\%) apresentou recidiva com 12 meses, sendo também realizada totalização com boa evolução. Os dois pacientes da cordectomia e da near-total não apresentaram recidivas até a última avaliação.

Vinte e sete pacientes (90\%) permaneciam vivos e sem doença até o final deste estudo e $24(80 \%)$ com preservação da voz.

\section{DISCUSSÃO}

Embora a cirurgia conservadora da laringe tenha sido cada vez mais enfatizada nos últimos anos, os princípios cardinais para esse procedimento já datam desde a segunda metade do século XIX e até meados do século XX, com os trabalhos pioneiros de Solis-Cohen ${ }^{17}$ da dissecção subpericondral, de Gluck e Sorensen ${ }^{17}$ da hemilaringectomia vertical, de Leruoux-Robert ${ }^{17}$ da ressecção frontolateral e de Ogura $^{18}$ da ressecção supraglótica.

A permanência de uma via aérea sem necessidade de canulação, com uma função satisfatória para deglutição e fonação, e a ressecção completa da lesão maligna são os objetivos principais da cirurgia conservadora ${ }^{1,2,4-8,15,17}$. 
Entretanto, para que possa realizar este procedimento de uma maneira mais funcional e com os princípios oncológicos adequados, nos últimos anos alguns autores têm preconizado alguns princípios básicos como adequado mapeamento da lesão através de estudos endoscópicos e por imagens, o não-comprometimento dos resultados terapêuticos para manutenção da função, o status nutricional aceitável, as boas funções clínicas, notadamente cardiopulmonar, e adequada reconstrução, a qual é variável, dependendo da extensão da ressecção e do local da lesão ${ }^{6,17,19,20,21}$.

Em relação a esse último item, para que a reconstrução ofereça uma função fonatória adequada com proteção da via aérea e deglutição normal, Spector ${ }^{17}$ recentemente preconizou alguns critérios: um adequado lúmen para via aérea, geralmente de 4 a $6 \mathrm{~mm}$, no nível da glote; manutenção da função de uma aritenóide e parte da prega vocal; manutenção de uma adequada rigidez lateral do restante da laringe que é mantido; manutenção da elasticidade longitudinal para que se realize a elevação da laringe durante a deglutição; manutenção de um sincronismo adequado da deglutição e respiração através da utilização de retalhos cutâneos, miocutâneos ou mesmo interposição de tecido cartilaginoso.

Segundo esses princípios é que as técnicas conservadoras da ressecção supracricóidea da laringe cada vez mais nos últimos anos têm sido solidificadas como a ressecção supracricóidea com crico-hioidepexia, uma das mais recentes conquistas dentro das indicações da realização de cirurgias mais funcionais ${ }^{22-24}$. Desde que bem indicado para os tumores glóticos selecionados, transglóticos e carcinomas supraglóticos endolaríngeos, esse procedimento tem demonstrado índices de sobrevida de $84 \%$ a $95 \%$ e com manutenção satisfatória da função laríngea e sem necessidade de canulação em até $100 \%{ }^{6,7,25-27}$. Os maus resultados citados por alguns autores se devem principalmente a não seguirem os preceitos oncológicos necessários, já que esse tipo de procedimento técnico é totalmente contra-indicado quando há fixação da corda vocal, extensão infraglótica com comprometimento da cartilagem cricóide, invasão do osso hióide e pericôndrio externo da cartilagem tireóide, invasão do espaço pré-epiglótico com protuberância na valécula e/ou na membrana tiro-hióidea ${ }^{28}$.

Dos quatro pacientes em que realizamos esse procedimento, em três houve boa evolução sem vigência de doença no tempo de avaliação realizada entre um e três anos e com função adequada, apresentando deglutição satisfatória, via aérea pérvia e sem necessidade de canulação em dois deles. Mais recentemente, alguns autores têm demonstrado fatores de risco para aspiração como idade acima de 65 anos, ressecção da cartilagem aritenóide, história prévia de refluxo gastroesofágico, transecção ou ligadura de $12^{\mathrm{o}}$ par craniano, laríngeo superior e inferior, preconizando, assim, a realização da gastrostomia endoscópica, nos primeiros dias de pós-operatório ${ }^{28}$. O paciente da nossa série que apresentou recidiva tratava-se de uma lesão T3 de glote com invasão para a região supraglótica sendo indicada totalização.

No que se refere à laringectomia supraglótica, esta permanece como procedimento cirúrgico standard com uma boa segurança oncológica, desde que bem indicada, como demonstraram De Santo ${ }^{29}$, Lutz et al. ${ }^{30}$, que apresentaram uma recidiva local menor que $2 \%$ em pacientes submetidos a essa técnica. Outros, como Stafin ${ }^{31}$, demonstraram em revisão extensa da literatura em 15.000 casos, uma sobrevida de $75 \%$ em cinco anos quando este procedimento foi bem indicado.

Outro dado importante em relação a essa técnica é que a mesma apresenta bons resultados funcionais, oferecendo uma boa deglutição com respiração pela via aérea normal e qualidade de voz satisfatória. Para que se possa obter melhor qualidade dos parâmetros funcionais, é importante levar em consideração a extensão da ressecção supraglótica, como tem sido demonstrada por alguns autores ${ }^{10,12,33,34}$. Assim, cada vez mais se tem dado importância para a manutenção do seio piriforme, cartilagem aritenóide e base da língua para se obter uma melhor função da deglutição ${ }^{32}$. Entretanto, a manutenção dessas estruturas muitas vezes não é obtida para que não se comprometa os princípios oncológicos, o que levaria, conseqüentemente, a uma menor qualidade de deglutição. E isso ficou bem demonstrado na série de Rademarker et al. ${ }^{10}$ que, analisando 37 pacientes submetidos a extensões diferentes de ressecções supraglóticas, demonstraram que o grupo de pacientes em que foi realizada somente ressecção supraglótica simples apresentou $70 \%$ de ingestão normal e com uma média no 26ㅇ dia. Já no grupo de pacientes em que houve também ressecção do seio piriforme e cartilagem aritenóide, somente $27 \%$ em média apresentaram ingestão normal no pós-operatório, com média no 54을. dia. E no grupo em que se associou também ressecção de base de língua, a média de deglutição ocorreu somente em 121 dias.

Este fato ficou bem evidente em nosso estudo, pois em dois pacientes submetidos a esse procedimento associado à ressecção da cartilagem aritenóide e parte da base da língua, não conseguiram deglutir até o 45 e $60^{\circ}$ dias de pós-operatório. Mesmo assim, após esse tempo, apresentaram dificuldades de deglutição com aspirações intermitentes, o que fez com que retardasse a decanulização, e em um deles ainda foi necessária a realização da totalização da laringectomia, devido a pneumonias de repetição. Os dois pacientes restantes, em que não houve ressecção dessas estruturas, tiveram boa ingestão oral a partir do $12^{\circ}$ e $15^{\circ}$ dias com decanulização nesse tempo e sem intercorrências.

Em revisão feita por Sartore e Swenn ${ }^{35}$ em 674 casos em que foram submetidos à ressecção supraglótica, $83,5 \%$ dos pacientes decanulizaram nos primeiros 60 dias, com ingestão via oral satisfatória e sem aspiração nos primeiros 30 dias, em 94,5\% dos casos. Além disso, os pacientes apresentaram qualidade de voz satisfatória, com presença de rouquidão em $78 \%$ dos casos.

Mais recentemente, alguns autores têm preconizado a ressecção da lesão supraglótica por via endoscópica, demonstrando as vantagens desse procedimento para melhor reabilitação, seja pelo não comprometimento da via aérea, mesmo que temporária, além de uma melhor deglutição pelo não comprometimento do nervo laríngeo 
superior e também pela não secção da musculatura suprahióidea. Além disso, o defeito supraglótico se cicatriza completamente por fibrose secundária e epitelização, o que favorece uma cicatrização mais favorável e que funcionaria como uma válvula supraglótica ${ }^{12,36,37}$. Entretanto, a realização desse procedimento por via endoscópica tem que ter uma indicação bem precisa para não comprometer os princípios oncológicos. Assim, o ideal é que se realize em lesões T1 e T2 que estejam presentes na epiglote suprahioidiana, pregas ariepiglóticas e prega vestibular, já que são locais de melhor visualização do lúmen distal do laringoscópio devido à posição anatômica mais perpendicular ${ }^{12}$, 36, 37 . Em nossa série, por serem lesões mais extensas e estarem localizadas na epiglote infra-hioidiana ou mesmo na falsa prega vocal, houve contra-indicação por via endoscópica.

Para outros autores, em lesões T2 e T3 com invasão dos espaços para glóticos e pré-epiglóticos, a indicação para terapia endoscópica só teria sentido desde que se realizasse radioterapia pós-operatória, já que a ressecção pela via endoscópica seria considerada uma biopsia excisional de margens estreitas e, assim, não removeria de uma maneira adequada o espaço pré-epiglótico e para-epiglótico ${ }^{38,39,40}$.

Dentro ainda das várias indicações da laringectomia parcial, a ressecção vertical total ou parcial tem sido utilizada cada vez mais nos últimos anos desde que tenha sua indicação precisa para não comprometer os princípios oncológicos. Essas lesões são tumores T2 de prega vocal unilateral, T3 confinado a uma prega vocal e que não comprometa a comissura anterior, T2 supraglótico ocupando somente uma falsa prega $a^{5,10,14,15,41}$. Dentro dessas indicações é que os oito pacientes de nossa série foram submetidos a esse tipo de procedimento com baixa morbidade traduzida somente por um caso de fístula faringocutânea, o qual teve boa evolução com tratamento conservador $(12,5 \%)$. Fato esse também observado por outros autores ${ }^{5,14,15}$.

A grande vantagem desse procedimento é que, além de ser radical em relação ao tumor, ele faz com que a maioria dos pacientes possa ser reabilitada com voz rouca, porém bem satisfatória e com decanulização geralmente precoce em $80 \%$ a $100 \%$ dos $\operatorname{casos}^{5,10,14,15}$. E isso ficou bem demonstrado em nosso estudo, já que $87,5 \%$ dos pacientes foram decanulados em três semanas em média e com voz e respiração bem aceitáveis. Outro fato que também estimula o emprego dessa técnica é que a ingestão via oral é bastante satisfatória, geralmente normal por não apresentar quase aspiração com taxa variando entre $86 \%$ e $100 \%$ dos casos ${ }^{10,14,15,42}$. Esses dados também são compatíveis com nosso estudo, pois todos os pacientes ingeriram dieta geral por via oral adequadamente.

Os resultados funcionais da hemilaringectomia vertical em relação à laringectomia supraglótica parecem ser melhores, principalmente no que se refere à ingestão oral adequada sem aspiração como demonstraram Rademarker et al. $(1995)^{10}$. Em sua série de pacientes, submetidos à hemilaringectomia vertical, 91,6\% conseguiram ingerir dieta semelhantes ao pré-operatório, com tempo médio de um mês, o que foi superior ao grupo de pacientes com ressecção supraglótica em que a ingestão via oral normal variou de $25 \%$ a $74 \%$, dependendo da extensão da ressecção. Esses dados também foram similares no nosso estudo, embora com um número pequeno de casos, em que foi demonstrado que os resultados funcionais foram melhores para o grupo da hemilaringectomia vertical. Isso se deve ao fato de que a hemilaringectomia não afeta a epiglote e prega ariepiglótica contralateral, as quais servem para desviar o bolo alimentar lateralmente ao redor da via aérea. Esse fator não é observado na ressecção supraglótica que, pela ressecção total da epiglote e prega ariepiglótica, faz com que o bolo alimentar migre para a linha média e lateral. Assim, o paciente deve aprender uma nova maneira de deglutição para evitar a aspiração, fato esse nem sempre fácil, já que na maioria das vezes esses pacientes em nosso meio apresentam condições socioeconômicas e culturais bastante precárias.

Outra técnica utilizada até os dias de hoje, desde a sua padronização por Leroux-Robert em $1953^{43}$, é a laringectomia frontolateral. É um procedimento de grande valia, principalmente por ser o mais conservador possível, com mínima mortalidade, além de baixa morbidade $2,13,44,45$. Isso ficou bem evidente em nossa série, pois dos 12 pacientes que foram submetidos a esse procedimento, somente um $(8,3 \%)$ apresentou complicação traduzida por um pneumotórax, facilmente resolvido pelo tratamento preconizado. Além disso, essa técnica oferece aos pacientes boa qualidade vocal e decanulização precoce no pósoperatório.

Entretanto, torna-se necessário que tenha indicação precisa para que os princípios oncológicos não sejam comprometidos. Assim, as lesões T1 de prega vocal são a grande indicação, mesmo que comprometam a comissura anterior, mas com prega vocal móvel. Isso ficou bem evidente em nosso estudo, estando os 12 doentes vivos e com boa qualidade de vida até o final dessa avaliação.

Muitos autores, entretanto, têm preconizado para tumores $\mathrm{T} 1$ de prega vocal a radioterapia, por oferecer melhor qualidade de voz, ser menos onerosa, além de evitar a hospitalização e o trauma cirúrgico, com sobrevida semelhante ao pró-cirúrgico $(85 \% \text { a } 100 \%)^{2,13}$.

Em revisão recente da literatura, Mendenhal et al. em $1994^{2}$, em 481 pacientes submetidos à radioterapia para tumores glóticos T1, demonstraram $84 \%$ de controle local da doença. Apesar desses resultados, outros não são favoráveis a esse tipo de terapêutica, já que a mesma também oferece desvantagens, como o paciente ficar ausente de suas atividades para a realização desse procedimento de seis a oito semanas e complicações como condrites, estenose e indução de um segundo tumor primário, posteriormente que, embora de baixa incidência, não ultrapassando $2 \%$ a $5 \%$, pode ser bem comprometedor ao paciente ${ }^{13}$.

O único paciente no qual realizamos a laringectomia near-total apresentava estádio T3N0 de prega vocal, tendo o mesmo boa evolução e sem doença residual na última avaliação realizada. A maioria dos autores que indica esse tipo de procedimento, também o faz em estádios 
T3 e T2, com resultados funcionais satisfatórios no que se relaciona à deglutição, voz e respiração na maioria dos pacientes e com sobrevida média de $78 \%{ }^{11,46}$. Mais recentemente, com o advento das próteses vocais, proporcionando uma reabilitação bastante satisfatória, fez-se com que esse tenha indicação bem limitada ${ }^{47}$.

O paciente restante, que foi submetido à cordectomia, encontra-se vivo e sem recidiva na última avaliação realizada. Quando se indica esse tipo de ressecção, geralmente é para tumores iniciais localizados na porção mediana da prega vocal, demonstrando índices de cura supe- riores a $90 \%$ na maioria das séries e com boa qualidade de vida $^{11,48}$. Atualmente, esse tipo de procedimento tem sido quase exceção, já que esse tipo de ressecção tem sido realizado por via endoscópica ou mesmo sendo indicada a radioterapia, já que esta proporciona sobrevida semelhante e com melhor qualidade vocal e baixa morbidade.

Assim, concluímos que os casos avançados e selecionados são passíveis de serem curados, mantendo-se a função da laringe, e que a hemilaringectomia vertical proporciona melhor reabilitação quanto à deglutição e fonação, quando comparada à ressecção supraglótica.

\begin{abstract}
Background: This is of a retrospective study of the results obtained from partial laryngectomy for epidermoid carcinoma. Methods: Twenty-eight males (93.5\%) and 2 females (6.5\%), between 42 years and 66 years old (mean age, 58 years). The surgical procedure varied in accordance to the site, extension and stage of the tumor. The technique most used was the frontolateral laryngectomy, performed on 12 patients (40\%), followed by the vertical laryngectomy on 8 patients (26.6\%), the supraglottic laryngectomy on 4 patients (13.3\%), supracricoid laryngectomy on 4 patients (13.3\%), near total laryngectomy on 1 patient (3.3\%) and chordectomy on 1 patient (3.3\%). Results: An early postoperative assessment showed that 3 patients (10\%) had complications. Two of them had pharyngocutaneous fistulas that responded well to conservative treatment and the remaining patient had pneumothorax, which improved after thoracic drainage. Deglutition assessment revealed that 5 patients $(16.6 \%)$ presented difficulty in oral ingestion as a result of recurrent aspirations that occurred at periods that varied between 15 days to 180 days (average 180 days) and caused recurrent pneumonia. The only remaining option was total laryngectomy. The next assessment was performed after a period that varied between 6 months to 125 months (mean of 29 months) and revealed that 4 patients (13.3\%) had recurrence in the same region. Two of these patients underwent vertical laryngectomy, 1 patient had a frontolateral laryngectomy and the remaining patient underwent supracricoid laryngectomy. All patients underwent total laryngectomy and/or radiotherapy, but the disease caused the death of 2 patients. Twenty four patients (84\%) demonstrated that their voice was preserved and at the end of the assessment, $90 \%$ of the patients were alive and without any signs of the disease. These patients had a far better quality of life as they presented fairly satisfactory voice and oral ingestion. Conclusion: The conservative surgery of the larynx provided good disease control and increased the patient's life span.
\end{abstract}

Key Words: Larynx; Carcinoma; Laryngectomy.

\title{
REFERÊNCIAS
}

1. Misiolek M, Ziora D, Oklek K et al. Evaluation of upper airway obstruction after partial laringectomies by radiological method and flow-volume loop analysis. The Journal of Laringology and Otology. 1994; 108:954-956.

2. Mendehall WM, Parsons JT, Stringer SP et al. Management of T1s and T2 squamous cell carcinoma of the glottic larynx. American J. Otolaringology. 1994; 15:250-257.

3. Delgaudio M, Flemming JJ, Esclamado RM et al. Hemilaringectomy for glottic carcinoma after irradiation therapy failure. Arch Otholaringol Head and Neck Surg. 1994; 120:959-963.

4. Nibu K, Kamata S, Kawabata K et al. Partial laringectomy in the treatment of radiation failure of early glotic carcinoma. Head and Neck. 1997; 19:116-120.
5. Lydiatt WM, Shah JP, Lydiati KM. Conservation surgery for recurrent carcinoma of the glottic larynx. Am. J. Surg. 1996; 172:662-664.

6. Laccourrey O, Brasnu O, Meritte-Dransy A et al. Cricohioidopexy in selected infrahioidoepiglotic carcinoma S senting with patological preepiglotic space invasion. Arch Otolaryngol. Head and Neck Surg. 1993; 119:881-886.

7. Chevalier D, Watelet J, Darras JA et al. Supraglotic hemilaryngopharyngectomy plus radiation for the treatment of early lateral margin and pyriform sinus carcinoma. Head \& Neck. 1997; 19:1-5.

8. Myers EN, Alui A. Management of carcinoma of the supraglottic larynx: Evolution, current concepts and future trents. Laryngoscope. 1996; 106:559-567. 
9. Barzan L, Comoretto R. Hemipharyngectomy and hemilaryngectomy for pyrifor sinus cancer reconstruction with remaining larynx and hypopharinx with tracheostomy. Laryngoscope. 1993; 103:82-86.

10. Rademarker AW, Logemann JA, Pauloski BR et al. Recovery of post operative swallowing in patients undergoing partial laryngectomy. Head \& Neck. 1993; 15:325-334.

11. Zanaret M, Giovani A, Gras R et al. Near total laryngectomy with epiglotic reconstructions: long term results in 57 patients. Am. J. Surg. 1993; 18:419-425.

12. Zeitels S. Surgical management of early supraglottic cancer. Otolaryngologic Clinics of North America. 1997; 30:59-78.

13. Osguthorpe D, Putney F.J. Open surgical management of early glottic carcinoma. Otolaryngologic Clinics of North America. 1997; 30:87-99.

14. Kooper DP, Van Den Broek P, Manni JJ et al. Partial vertical laryngectomy for recurrent glottic carcinoma. Clinic Otolaryngologic. 1995; 20:167-170.

15. Danilidis J, Nikolaov A, Foutzilas G et al. Vertical partial laryngectomy: our results after treatment 81 cases of T2 and T3 laryngeal carcinomas. The Journal of Laryngologyc and Otology, 1992; 106:349-352.

16. Vincentiis M, Minni A, Gallo A et al. Supracricoid partial laryngectomies: oncologic and functional results. Head \& Neck. 1998; 20:504-509.

17. Spector JG. Conservation surgery for laryngeal cancer. Amm Overview. $4^{\text {th }}$ Conference of Head and Neck Cancer. Proceedings, 1996; 316-321.

18. Ogura JH, Jurema AA, Watson RK. Partial laryngectomy and neck dissection for pyriform sinus cancer. Laringoscope. 1960; 70:1399-1417.

19. Pearson BW. Subtotal laryngectomy. Laryngoscope. 1981; 91:1904-1907.

20. Spector JG, Sessions DG, Emani B et al. Squamous cell carcinoma of the aryepiglottic fold. Terapeutic results and long term follow up. Laryngoscope, 1995. 105:734-746.

21. Spector JG, Sessions DG, Emani B et al. Non randomized comparison of therapeutic modalities and lon term results. Laryngoscope, 1995; 105:397-406.

22. Laccourreye H, Laccourreye O, Weinsten G et al. Supracricoid laryngectomy with cricohyoidoepiglotticpexy for glottic carcinoma. Ann. Otol. Rhinol. Laryngol. 1990; 99:421-426.

23. Laccourreye H, Ross J, Brasnu D et al. Extendes supracricoid partial laringectomy with tracheocricohyoidoepiglotopexy. Acta Otolaringl. 1994; 114:669-74.

24. Laccourreye O, Weinstein G, Brasnu D et al. A clinical trial of continuous cysplatin-fluourouracil induction chemotherapy and supracricoid partial laringectomy for glottic carcinoma classified as T2. Cancer. 1994; 74:2782-2788

25. Pech A, Cannoni M, Giovanni A et al. La necessaire selection techinic chirurgicales dans cancers du larynx. Ann. Otolaryngol. Chir. Cervicofac. 1986; 103:565575.

26. Piquet JJ, Darras JA, Burny A et al. La chirurgie reconstrutive du larynx: Resultantes carcinologiques et funtionels. J. Fr. Otorhhinolaryngol. 1984; 33-215-220.
27. Piquet JJ, Chavalier D. Subtotal laryngectomy with cricohyoidoepiglotopexy for the treatment of extended glottic carcinoma. Am. J. Surg. 1991; 162:357-361.

28. Brasnu D, Weinsten G, Lacorried O. For endolaryngeal carcinoma. A review of the surgical procedures, oncological and functional indications and results. Proceedings. $4^{\text {th }}$ International Conference on Head and Neck Cancer. 1996. p. 326-332.

29. DeSanto LW. Cancer of the supraglottic larynx. Otolaryungol. Head and Neck Surg. 1985; 93:705-711.

30. Lutz CK, Johnson JT, Wagner RL. Supraglottic cancer: patterns of recurrence. Amm; Otol. Rhinus. Laringol. 1990; 99:12-17.

31. Staffieri A, Miani C, Pedoce E. Supraglottic laryngectomy today. In: Smee R, Bridger P. (eds.): Laryngeal Cancer. Proceedings of the $2^{\text {nd }}$ World Congress of Laryngeal Cancer. Amsterdan, Elsevier, 1994; p.71-475.

32. McConnel FMS, Mendelsohn MS, Lage EE, Mann JA. Manofluorography of deglutition after supraglottic laryngectomy. Head and Neck Surg. 1987; 9:142-150.

33. Kirchner JA. What have whole organs sections contributed to the treatment of laryngeal cancer? Ann. Otol. Rhinol. Laryngol. 1989. 98:661-667.

34. Sato K, Kurita S, Irano N. Location of the pre-epiglottic space and its relationship to the paraglottic space. Ann. Otol. Rhinol. Laryngol. 1993; 102:930-934.

35. Sartoris A, Succo G. Tratamento chirurgico conservativo di tumori souraglottici della laringe: Studio multicentrico sui risultati funzionali a distanza. In chirurgia funzionali della laringe. Statto attuale della arte. Atti del LXXX Congresso Nationali - Cap. XI, 339-351, Udine, 1993.

36. Zeitels SM, Koufmann JA, Davis LK et al. Endoscopic treatment of supraglottic and hypopharynx cancer. Laringoscope, 1994; 104:71-78

37. Shapiro J, Zeitels SM, Fried MF. Laser surgery for laryngeal cancer: operative techniques in otolaryngology. Head and Neck Surgery. 1992; 3:84-92.

38. Steiner W. Results of curative laser microsurgery of laryngeal carcinoma. Am. J. Otolaryngol. 1993; 14:116121.

39. Mendenhall WM, Million RR, Cassissi MJ. Squamous cell carcinoma of the supraglottic larynx treates with radical radiation: analysis of treatment parameters and results. Int. J. adiat. Oncol. Biol. Phys. 1985; 10:22232230

40. Spalding CA, Krochak RJ, Seug SH et al. Radioterapeutic management of cancer of the supraglottis. Cancer. 1986; 57:1292-1298.

41. Kessler DJ, Trapp TK, Calcaterra TC. The treatment of the T3 glottic carcinoma with vertical partial laryngectomy. Archives of Otolaringology - Head and Neck Surgery, 1987; 113:1196-1199.

42. Scolla B, Veja MF, Martinez T et al. Extended hemilaringectomias - Proceedings $4^{\text {th }}$ International on Head and Neck Cancer, 1996; 333-338.

43. Leroux-Robert. La quirurgie conservatica par laringofissure on laringectomie partielle dan le cancer du laringe. Les Ann. D’Oto-laringe. 1957; 74:40-74.

44. Shah JP, Soo KC. Indicação da laringectomia parcial. In: Brandão LG, Ferraz AL, Eds. Cirurgia de Cabeça e Pescoço, $1^{a}$ edição, São Paulo, Rocco Editora, 1989, pp.261-265. 
45. Aprigliano F, Aprigliano FF. Laringectomias parciais - aspectos funcionais. In: Brandão LG, Ferraz AL. eds. Cirurgia de Cabeça e Pescoço, $1^{a}$ edição, São Paulo, Rocco Editora, 1989, pp.267-271.

46. Pearson BW. Surgical options in laringeal carcinoma. Proceedings $4^{\text {th }}$ International on Head and Neck Cancer, 1996, 309-315.

47. Costa CC. Reabilitação vocal do laringectomizado com prótese traqueoesofágica. Tese de Mestrado apresentada ao Curso de Pós-Graduação do Complexo Hospitalar Heliópolis - SP, outubro, 2000.
48. Silver CE, Valdez ME. Aspectos técnicos da laringectomia parcial. In: Brandão LG, Ferraz AL. eds. Cirurgia de Cabeça e Pescoço, $1^{a}$ edição, São Paulo, Rocco Editora, 1989, pp.273-285.

Endereço para correspondência:

Dr. José Luis Braga de Aquiino

Rua John Boyd Dunlop, S/N - Jardim Ipaussurama 13059-740 — Campinas-SP 